\title{
Longitudinal Results With Intratympanic Dexamethasone in the Treatment of Ménière's Disease
}

\author{
Maria Soledad Boleas-Aguirre ${ }^{\star}, \dagger$, Frank R. $\operatorname{Lin}^{\dagger}$, Charles C. Della Santina ${ }^{\dagger}$, Lloyd B. Minor ${ }^{\dagger}$, \\ and John P. Carey ${ }^{\dagger}$ \\ *Clínica Universitaria de Navarra, School of Medicine, University of Navarra, Pamplona, Spain \\ †The Johns Hopkins Hospital, The Johns Hopkins University, Baltimore, Maryland, U.S.A.
}

\section{Abstract}

Objective-To assess patient satisfaction with vertigo control using intratympanic (IT) dexamethasone $(12 \mathrm{mg} / \mathrm{mL})$ for medically refractory unilateral Ménière's disease.

Study Design-Retrospective study.

Setting-Tertiary referral neurotology clinic.

Patients-One hundred twenty-nine subjects diagnosed with unilateral Ménière's disease still having vertigo despite medical therapy.

Intervention-IT dexamethasone injections as needed to control vertigo attacks.

Main Outcome Measure-A Kaplan-Meier time-to-event method was used to determine the rate of "survival," meaning sufficient satisfaction with vertigo control that the subject did not wish to have subsequent ablative treatment. "Failure" was defined as poor control and the choice to proceed to ablative treatment.

Results-Acceptable vertigo control ("survival") was achieved in 117 (91\%) of 129 subjects. Vertigo control required only one dexamethasone injection in 48 (37\%), 2 injections in 26 (20\%), 3 injections in $18(14 \%)$, and 4 injections in $10(8 \%)$. More than 4 injections were needed in 15 subjects $(21 \%)$. Of 12 failures (9\%), 9 occurred within 6 months of the first IT dexamethasone injection. Follow-up data for 2 years were available for 96 subjects. Of these, $87(91 \%)$ had vertigo control with IT dexamethasone, of whom 61 (70)\% required no further injections after 2 years, $23(26 \%)$ continued to receive IT dexamethasone injections, and $3(3 \%)$ chose IT gentamicin treatment.

Conclusion-IT dexamethasone injection therapy on an as-needed outpatient basis can provide vertigo control that is satisfactory in patients with Ménière's disease. The Kaplan-Meier method addresses the need for an outcome measure suited to repeated treatments and variable lengths of follow-up. However, due to the retrospective nature of this study, the presence of bias caused by loss of subjects from follow-up cannot be ruled out.

\section{Keywords}

Intratympanic; Dexamethasone; Gentamicin; Ménière's disease; Endolymphatic hydrops; Vertigo 
Intratympanic (IT) therapies are growing in popularity in the management of vertigo in Ménière's disease. The success of IT gentamicin has been examined in a number of series and summarized in several meta-analyses (1-3). Although vertigo control is achieved in more than $85 \%$ of the subjects in these series, hearing loss and posttreatment disequilibrium remain significant concerns. The success of "titration protocols" for IT gentamicin has inevitably led to the attempt to use non-ablative IT steroids to control vertigo attacks in Ménière's disease in a similar way. A PubMed search found 29 articles related to IT steroid treatment for Ménière's disease over the last decade.

IT steroids may have an anti-inflammatory effect on the labyrinth as suggested by the beneficial response in inner ear disorders with likely immune causes (4). In addition, recent in vitro physiology studies suggest that steroid perfusion of labyrinthine tissues can affect sodium and fluid transport (5). Trune et al. (6) have identified a mouse with progressive stria vascularis dysfunction and hearing loss in which steroids with mineralocorticoid effects ameliorate the inner ear dysfunction. Therefore, it is also possible that the effects of IT steroids in Ménière's disease include an ion or water transport mechanism.

One of the first reports regarding the effects of IT steroids for Ménière's disease showed $80 \%$ improvement in vertigo and a 74\% reduction in tinnitus in 61 patients (7). The use of IT and intravenous corticosteroids showed short-term control of vertigo in $96.4 \%$ and an improvement in hearing loss in $67.9 \%$ of 28 patients in Shea's stages I to III of Ménière's disease (8). These and other preliminary results led Silverstein et al. (9) to conduct a prospective, randomized, double-blind study to compare IT dexamethasone with sodium hyaluronate versus saline with sodium hyaluronate. IT dexamethasone did not show benefit over placebo for improving vertigo, hearing loss, or tinnitus in this small study of subjects with late-stage Ménière's disease (Shea's stage IV). However, other authors have reported good vertigo control rates in the short term after IT steroid treatment. Sennaroglu et al. (10) found that $72 \%$ of their subjects had satisfactory control of vertigo, $16 \%$ had improvement of hearing, and only $8 \%$ had worsening of hearing after IT dexamethasone treatment. In a retrospective study, Barrs (11) reported control of vertigo with one or more courses of IT injections of dexamethasone in $47 \%$ of patients with intractable Ménière's disease. A recent prospective, placebo-controlled study with 2 years' follow-up demonstrated complete control of vertigo in $82 \%$ of those with IT steroid treatment versus 57\% with placebo treatment (12).

Despite these mixed preliminary results, IT steroid therapy appears to be increasing in clinical practice. This is no doubt influenced by the convenience and ease of repeating these officebased treatments. Moreover, minimal side effects have been reported related to the IT steroid therapy (mainly tympanic membrane perforation and inflammatory middle ear changes). The current study was conducted to determine if one or more IT steroid injections could provide sufficient relief from vertigo or disequilibrium in Ménière's disease that ablative treatments were subsequently avoided. For that purpose, we reviewed records from the previous 8 years in an academic neurotology practice for subjects who were patients with unilateral Ménière's disease that was unresponsive to medical therapy. The subjects were offered ablative therapy with IT gentamicin, but they were given the option of IT dexamethasone as a nonablative treatment first. We sought 3 important outcome measures: 1) the number and dates of IT dexamethasone injections, 2) the number of subjects who abandoned IT dexamethasone in favor of ablative treatments and when they did so, and 3) the number who continued with IT dexamethasone injections after 2 years from the first injection.

\section{MATERIALS AND METHODS}

This is a retrospective study from November 1997 to May 2006 including patients who were seen in clinical consultation at the Department of Otolaryngology-Head and Neck Surgery at 
the Johns Hopkins University School of Medicine (Baltimore, MD, USA) and were diagnosed with definite unilateral Ménière's disease by the 1995 criteria of the American Academy of Otolaryngology - Head and Neck Surgery (AAO-HNS). According to these criteria, definite unilateral Ménière's disease must meet the following criteria: 1) 2 or more definitive spontaneous episodes of vertigo of 20 minutes or longer, 2) audiometrically documented hearing loss on at least 1 occasion, 3 ) tinnitus or aural fullness in the treated ear, and 4) other causes excluded (13). None of these subjects responded adequately to dietary or diuretic treatments, although sodium restriction and diuretic therapy were continued if there was a history of some beneficial response. The attending physician explained to the patient the pros and cons of different therapeutic options: IT injection of steroids, IT injection of gentamicin, endolymphatic sac surgery, vestibular neurectomy, and labyrinthectomy. Patients were offered IT dexamethasone treatment as an option that might provide temporary relief of vertigo without destroying vestibular function. Of 132 patients thus counseled, 3 opted for IT gentamicin immediately, and 129 opted to try IT dexamethasone before proceeding to IT gentamicin. The latter 129 are the subjects of this study. This study was a review of existing clinical data with patient identifiers removed. It qualified for exemption from an institutional review board protocol based on US Department of Health and Human Services criteria 45 CFR 46.101(b4). The Johns Hopkins Medicine Institutional Review Board made the determination that the study qualified for this exemption.

The IT steroid injection procedure was performed in the outpatient clinic under a binocular microscope after informed consent was obtained from the patient. With the patient supine and the head turned to the opposite side, a drop of phenol was used to induce local anesthesia on the anterior aspect of the tympanic membrane. The dexamethasone $(12 \mathrm{mg} / \mathrm{mL})$ solution was loaded into a $1-\mathrm{mL}$ tuberculin-type syringe with a long 25 -gauge needle attached on it. One needle hole was made in the anesthetized area for air to escape during the middle ear injection. A second hole was made for the injection, and the drug solution was infused into the middle ear space in a quantity (usually $0.4-1.0 \mathrm{~mL}$ ) sufficient to fill the space or at least cover the round-window niche. After the injection, the head was placed slightly lower than the body, and the subject was instructed to lie in the supine position with the head turned 45 degrees toward the contralateral side, keeping the treated ear up for 30 minutes.

In the following clinical appointments (at 1- to 4-month intervals), vertigo control was assessed by the subject's report of the frequency and severity of any vertigo attacks that occurred since the last treatment. If there were no vertigo attacks reported, then no further therapy was recommended at that time. If there were any vertigo attacks but the patient was satisfied with a reduction in the frequency or severity compared with what they had experienced before the IT dexamethasone injection, then the subject was offered another IT dexamethasone treatment. However, if the subject felt that the effect of the last IT dexamethasone injection was negligible or unsatisfactory, then IT gentamicin injection or ablative surgery was recommended.

Measured data were the number and dates of dexamethasone injections that the subjects received during the period between their first injection and an administrative censor date when the analysis was initiated (May 9, 2006). For those who failed therapy before the censor date, we determined the same data as well as when the failure occurred. Records were also reviewed for evidence of persistent tympanic membrane perforations after injections.

In keeping with the AAO-HNS criteria calling for 2 years' follow-up for treatment studies in Ménière's disease (13), we also determined how many subjects remained on IT dexamethasone treatment after 2 years from their first injection and how many had chosen one of the other therapies mentioned. We classified subjects by AAO-HNS stage of Ménière's disease before IT dexamethasone treatment, and we assessed in each class the number of injections that the subjects had received. 


\section{Statistical Analysis}

A Kaplan-Meier time-to-event method was used to calculate the percentage of the subjects who had adequate control of their vertigo over time after initial IT dexamethasone injection ("survival") (14). Data for all subjects who did not fail treatment were censored administratively (i.e., censored due to constraints of when analysis was performed rather than being lost to follow-up) (15). Kaplan-Meier calculations with 95\% confidence intervals estimated with Greenwood's formula were performed using Stata 8.2 software (Stata Corp., College Station, TX, USA).

\section{RESULTS}

The Kaplan-Meier survival curve in Figure 1 demonstrates that 91\% (117 of 129) subjects were sufficiently satisfied with the course of their IT dexamethasone treatments that they did not choose to go on to a different therapy during the study period. All 12 of the subjects who selected another therapy selected IT gentamicin treatment. Previous to gentamicin, 5 of these 12 subjects received 1 IT dexamethasone injection, 3 subjects received 2 injections, 3 other subjects received 4 , and one subject received 8 . Nine of these 12 failed IT dexamethasone therapy within the 6 months of the first dexamethasone injection. Eight of these "early" failures had received 1 or 2 IT dexamethasone injections. One subject failed in the third year after receiving 4 IT dexamethasone injections. Two subjects failed in the fourth year following their first IT dexamethasone injections after they received a total of 4 and 8 dexamethasone injections, respectively.

Vertigo control required only 1 dexamethasone injection in 48 survivors (37\%), 2 injections in $26(20 \%), 3$ injections in 18 (14\%), and 4 injections in $10(8 \%)$. More than 4 injections were needed in 15 survivors $(21 \%)$. Table 1 summarizes the cumulative number of dexamethasone injections given to survivors by each year of follow-up. Most subjects received 1 to 3 IT dexamethasone injections.

Of the total 129 subjects, 96 had at least 2 years of follow-up. Nine of these subjects (9\%) chose to go on to ablative therapy with IT gentamicin treatment because of unsatisfactory control of their vertigo. They were considered to be IT dexamethasone treatment "failures." The remaining 87 survivors (91\%) with at least 2 years of follow-up found satisfactory vertigo relief with variable numbers of IT dexamethasone injections (Table 1, second column). Of these, 37 subjects ( $42 \%)$ required no further steroid injections than the first one, 21 (24\%) needed 2 injections, $11(13 \%)$ required 3 injections, 11 (13\%) required 4 injections, $3(3 \%)$ required 5 injection, $3(3 \%)$ required 6 injections, and $1(1 \%)$ required 7 injections as of the 2year follow-up point. For 87 subjects with follow-up beyond 2 years, 61 (70\%) did not require any further treatment, $23(26 \%)$ received more IT dexamethasone injections, and $3(3 \%)$ chose IT gentamicin treatment. Table 2 gives the cumulative number of injections received by subjects with each stage of Ménière's disease.

\section{DISCUSSION}

IT therapy for Ménière's disease has evolved since the introduction of "titration protocols" for IT gentamicin (16). The success of such intermittent, "as-needed" injections of gentamicin inevitably has led to the similar use of IT steroids. In such treatment schemes, the need for a second injection would constitute "failure" under the 1995 AAO-HNS criteria. Yet patients commonly choose to continue with repeated injection therapies instead of proceeding to surgically ablative ones. In the case of IT steroid injection, the elements underlying the choice to continue with repeated injections may be numerous, and some are difficult to measure. These elements may include positive effects of the steroid injection, including actual reduction in the frequency or severity of vertigo attacks, reduced aural pressure sensation, or even placebo 
effects. The decision may also be affected by the subject's unease with the risks of other therapies, for example, IT gentamicin, which carries a greater risk of hearing loss and imbalance. Other elements affecting the choice for repeated IT steroid injections may include the perception of ease of treatment on the part of the patient or bias on the part of the otolaryngologist. The ability to receive immediate intervention soon after the recurrence of vertigo may give the patient reassurance and a perception of control over their disease that ultimately relieves the psychological stress that accompanies and may exacerbate this unpredictable disorder. Whatever the physiological and/or psychological effects of IT steroid treatment may be in Ménière's disease, it seems unlikely that patients would persist with this treatment if they did not consider it to have some beneficial effect on their symptoms.

Despite these ambiguous motivating factors, each choice to receive an IT steroid treatment represents a discrete and unambiguous decision point that can be used for longitudinal, quantitative analysis. The Kaplan-Meier method is suited to the type of analysis in this study, in which subjects had variable durations of follow-up ranging from 6 months to 8 years. Failure time was defined in this analysis as the time from the first injection of IT dexamethasone to the time at which a patient elected to receive IT gentamicin. For those who did not fail IT dexamethasone treatment, censoring time was defined to be the time from the first injection of IT dexamethasone to the time of data analysis. All censoring times in our study are, therefore, considered to be administrative (i.e., censoring due to constraints of when the analysis was performed rather than a patient actually being lost to follow-up) (15). This appears to be a reasonable assumption because all patients with Ménière's disease seen at our institution are followed up in the clinic regularly and told promptly to call if they experience debilitating vertigo. This assumption would only be compromised if the patient elected to receive treatment elsewhere and did not inform his or her clinician at our institution or if a patient died before the time of data analysis.

The survival curve in Figure 1 demonstrates that $91 \%$ of all subjects "survived" (i.e., received satisfactory vertigo control) with IT dexamethasone treatments given as needed. Very long follow-up data from some subjects yielded a survival curve that remained fairly flat beyond 2 years. For those 97 subjects with follow-up data at or beyond 2 years, the remission rate (needing no further intratympanic treatments) was 70\%. Before the advent of intratympanic therapies, Silverstein et al. (17) studied a group of patients with Ménière's disease who had failed medical therapy and were offered endolymphatic sac surgery or vestibular neurectomy. Of their 50 patients who declined any surgery, $57 \%$ had complete control of vertigo after 2 years, and $70 \%$ had complete control after 8.3 years. These findings indicate a high rate of spontaneous remission of vertigo in Ménière's disease even in patients who initially were felt to have medically refractory disease. In the present study, it is tempting to conclude that IT dexamethasone treatment led to more rapid remission of Ménière's disease. However, only a prospective placebo-controlled study designed specifically to compare remission rates between gentamicin and palcebo treatment would justify this conclusion, but such a study can be very difficult to perform.

It may be that IT dexamethasone therapy only provides temporary relief of symptoms and needs to be repeated until spontaneous remission occurs. The need for repeated injections would seem to be a disadvantage of IT dexamethasone in comparison with IT gentamicin treatment. The costs of each injection and the burden of experiencing recurrent vertigo at somewhat unpredictable times must be considered in this comparison. However, our experience with IT gentamicin demonstrates that multiple gentamicin injections are not unusual with titration protocol. The number of IT gentamicin injections needed to produce evidence of unilateral hypofunction and to control vertigo was $2.8 \pm 1.3$, and later recurrent vertigo required more gentamicin injections in $22 \%$ of patients (18). The IT gentamicin titration protocol would appear to have an advantage over IT dexamethasone treatment in clustering mutiple injections 
at the time of initial treatment and decreasing the probability for needing subsequent injections. However, IT gentamicin treatment carries the risks of worsening the hearing loss in the treated ear and of causing disequilibrium $(1,2)$ because a reduction in vestibular function is likely the mechanism by which gentamicin achieves vertigo control $(19,20)$.

Our approach to patients with medically refractory unilateral Ménière's disease with IT therapy has evolved. We discuss these advantages and disadvantages of IT dexamethasone versus IT gentamicin. Intratympanic dexamethasone treatment is encouraged if the patient has frequent vertigo because the apparently temporary effect would likely only benefit the patient who has a high probability of having vertigo in the few months after the treatment. If a beneficial effect is felt by the patient, subsequent injections are offered for recurrent symptoms at intervals of 1 to 3 months. However, if unsatisfactory effect is perceived by the patient, we then recommend proceeding to IT gentamicin treatment. Conversely, IT gentamicin is encouraged immediately if the patient has rare but severe attacks.

The growing popularity of IT therapy necessitates a reevaluation of the criteria for evaluating effectiveness of treatments for Ménière's disease. The 1995 AAO-HNS criteria for evaluating treatment outcomes in Ménière's disease were designed to assess one-time, primarily surgical, treatments by comparing the average monthly number of attacks of vertigo in the 6 months before treatment with the same number 18 to 24 months after treatment (13). Repeated office treatments were not envisioned in the creation of these criteria. However, when the regimen of as-needed, repeated IT dexamethasone injections is considered as a whole, it compares favorably with surgical options by the AAO-HNS outcomes criteria because $87(91 \%)$ of subjects with 2 years or more of follow-up found satisfactory vertigo relief with variable numbers of IT dexamethasone injectons (Table 1).

However, we suggest that the Kaplan-Meier approach has 2 advantages over these historic criteria. First, Kaplan-Meier methods allow more efficient use of all available data. Rather than only looking at subjects who have had a minimum of 2 years of follow-up, the Kaplan-Meier approach uses all of the available data to model the survival function over time (i.e., the percentage of patients over time with adequate vertigo control during treatment). Using this approach, we can therefore observe how vertigo control with a repeated treatment like IT dexamethasone varies over time rather than simply looking at vertigo control at only a crosssectional time point. Second, the Kaplan-Meier approach can use a patient-centered and clinically relevant outcome measure ("failure" defined as a patient's choice to receive a different therapy). This measure is discrete and not subject to recall bias or poor record keeping of vertigo spells on the part of subjects. Moreover, using the number of vertigo attacks as the primary outcome measure for treatment success can be problematic. For example, although class B control may be acceptable to some patients who previously had very frequent or severe attacks, it may still be very debilitating for other patients. Here, we have instead used a patientcentered outcome measure defined as when a subject chose to receive ablative IT gentamicin because his or her vertigo attacks were sufficiently debilitating to his or her lifestyle so that he or she was willing to accept the risks of ablative therapy. This approach is also consistent with the current emphasis on patient-centered outcomes and clinical decision making (21).

Hearing outcomes were not quantitatively addressed in this study, but we did not observe significant hearing changes in the majority of our subjects after IT dexamethasone treatment. Arriaga and Goldman (22) found that only $33 \%$ had hearing improvement, and $20 \%$ had hearing deterioration after IT dexamethasone with hyaluronan. However, Hillman et al. (23) reported a $40 \%$ hearing improvement after IT dexamethasone. Garduno-Anaya et al. (12) also reported a subjective improvement in tinnitus (48\%), hearing loss (35\%), and aural fullness (48\%) in the dexamethasone group compared with the control group. The risk of tympanic membrane perforation appears to be very low in our study (1 instance, $0.8 \%$ ) and probably related to the 
large number of injections given in that case. This finding suggests that patients requiring very frequent IT steroid injections should consider switching therapy to IT gentamicin to limit the total number of injections. The present data will hopefully motivate a well-designed prospective trial of IT steroids for Ménière's disease in which both hearing and equilibrium outcomes would be assessed.

\section{REFERENCES}

1. Cohen-Kerem R, Kisilevsky V, Einarson TR, et al. Intratympanic gentamicin for Ménière's disease: a meta-analysis. Laryngoscope 2004;114:2085-91. [PubMed: 15564826]

2. Chia SH, Gamst AC, Anderson JP, et al. Intratympanic gentamicin therapy for Ménière's disease: a meta-analysis. Otol Neurotol 2004;25:544-52. [PubMed: 15241234]

3. Diamond C, O'Connell DA, Hornig JD, et al. Systematic review of intratympanic gentamicin in Ménière's disease. J Otolaryngol 2003;32:351-61. [PubMed: 14967079]

4. Parnes LS, Sun AH, Freeman DJ. Corticosteroid pharmacokinetics in the inner ear fluids: an animal study followed by clinical application. Laryngoscope 1999;109:1-17. [PubMed: 10399889]

5. Pondugula SR, Sanneman JD, Wangemann P, et al. Glucocorticoids stimulate cation absorption by semicircular canal duct epithelium via epithelial sodium channel. Am J Physiol Renal Physiol 2004;286:F1127-35. [PubMed: 14996671]

6. Trune DR, Kempton JB, Kessi M. Aldosterone (mineralocorticoid) equivalent to prednisolone (glucocorticoid) in reversing hearing loss in MRL/MpJ-Fas1pr autoimmune mice. Laryngoscope 2000;110:1902-6. [PubMed: 11081607]

7. Itoh A, Sakata E. Treatment of vestibular disorders. Acta Otolaryngol Suppl 1991;481:617-23. [PubMed: 1927485]

8. Shea JJ, Ge X. Dexamethasone perfusion of the labyrinth plus intravenous dexamethasone for Ménière disease. Otolaryngol Clin North Am 1996;29:353-9. [PubMed: 8860933]

9. Silverstein H, Isaacson JE, Olds MJ, et al. Dexamethasone inner ear perfusion for the treatment of Ménière's disease: a prospective, randomized, double-blind, crossover trial. Am J Otol 1998;19:196201. [PubMed: 9520056]

10. Sennaroglu L, Dini FM, Sennaroglu G, et al. Transtympanic dexamethasone application in Ménière's disease: an alternative treatment for intractable vertigo. J Laryngol Otol 1999;113:217-21. [PubMed: 10435127]

11. Barrs DM. Intratympanic injections of dexamethasone for long-term control of vertigo. Laryngoscope 2004;114:1910-4. [PubMed: 15510013]

12. Garduno-Anaya MA, Couthino DT, Hinojosa-Gonzalez R, et al. Dexamethasone inner ear perfusion by intratympanic injection in unilateral Ménière's disease: a two-year prospective, placebocontrolled, double-blind, randomized trial. Otolaryngol Head Neck Surg 2005;133:285-94. [PubMed: 16087029]

13. Monsell EM, Balkany TA, Gates GA, et al. Committee on Hearing and Equilibrium guidelines for the diagnosis and evaluation of therapy in Ménière's disease. Otolaryngol Head Neck Surg 1995;113:181-5. [PubMed: 7675476]

14. Kaplan E, Meier P. Nonparametric estimation from incomplete observations. J Am Stat Assoc 1958;53:457-81.

15. Szklo, M.; Nieto, F. Epidemiology: Beyond the Basics. Jones and Bartlett Publishers; Boston, MA: 1999.

16. Toth AA, Parnes LS. Intratympanic gentamicin therapy for Ménière's disease: preliminary comparison of two regimens. J Otolaryngol 1995;24:340-4. [PubMed: 8699599]

17. Silverstein H, Smouha E, Jones R. Natural history vs. surgery for Ménière's disease. Otolaryngol Head Neck Surg 1989;100:6-16. [PubMed: 2493618]

18. Minor LB. Intratympanic gentamicin for control of vertigo in Ménière's disease: vestibular signs that specify completion of therapy. Am J Otol 1999;20:209-19. [PubMed: 10100525]

19. Carey JP, Minor LB, Peng GC, et al. Changes in the three-dimensional angular vestibulo-ocular reflex following intratympanic gentamicin for Ménière's disease. J Assoc Res Otolaryngol 2002;3:430-43. [PubMed: 12486598] 
20. Lin FR, Migliaccio AA, Haslwanter T, et al. Angular vestibulo-ocular reflex gains correlate with vertigo control after intratympanic gentamicin treatment for Ménière's disease. Ann Otol Rhinol Laryngol 2005;114:777-85. [PubMed: 16285268]

21. Zerhouni EA. US biomedical research: basic, translational, and clinical sciences. JAMA 2005;294:1352-8. [PubMed: 16174693]

22. Arriaga MA, Goldman S. Hearing results of intratympanic steroid treatment of endolymphatic hydrops. Laryngoscope 1998;108:1682-5. [PubMed: 9818826]

23. Hillman TM, Arriaga MA, Chen DA. Intratympanic steroids: do they acutely improve hearing in cases of cochlear hydrops? Laryngoscope 2003;113:1903-7. [PubMed: 14603044] 


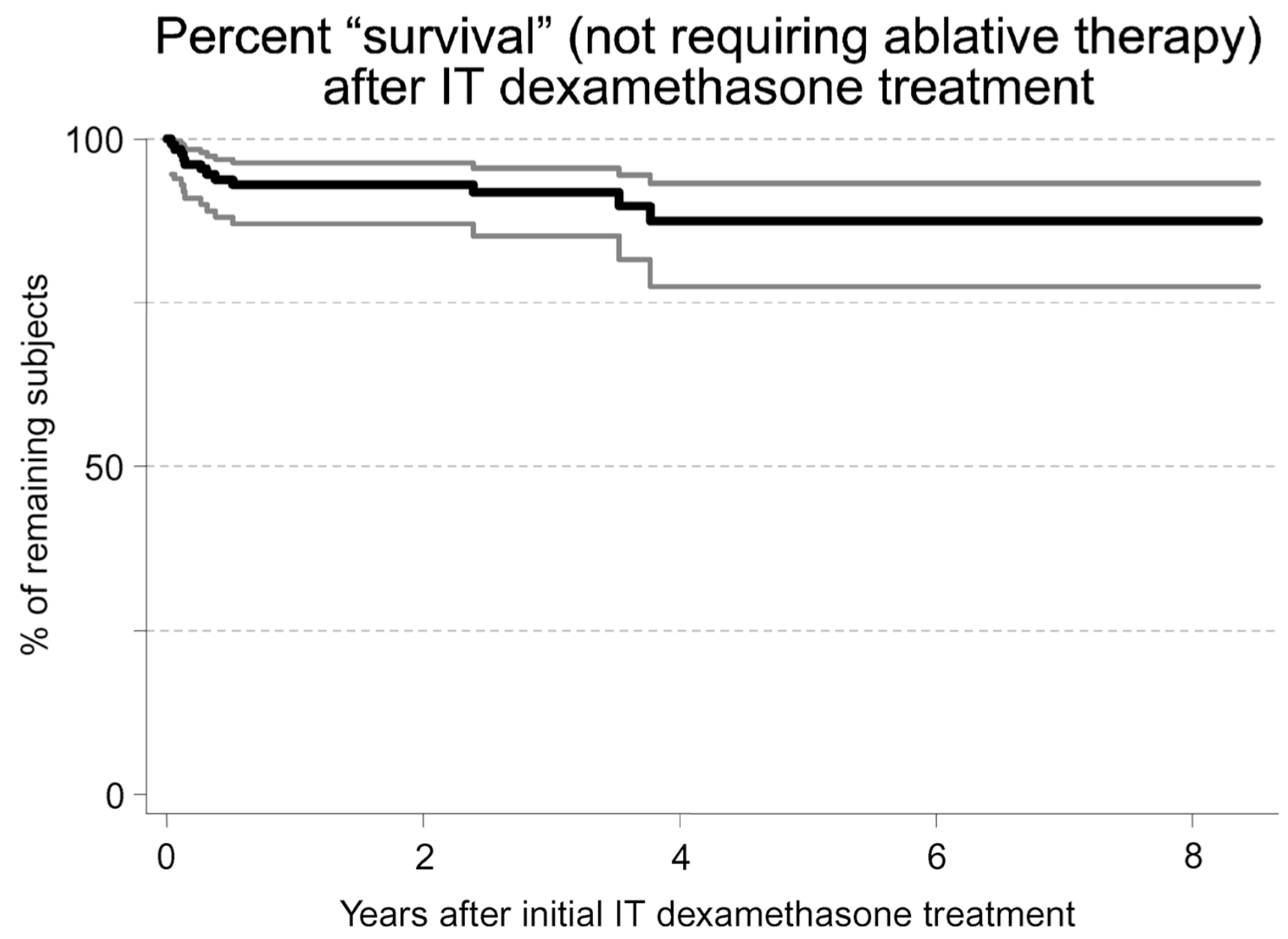

FIG. 1.

Kaplan-Meier Survival curve showing the percentage of subjects remaining on IT dexamethasone for vertigo control over time after initial injection. Black line indicates observed survival; gray lines, $95 \%$ confidence interval. 


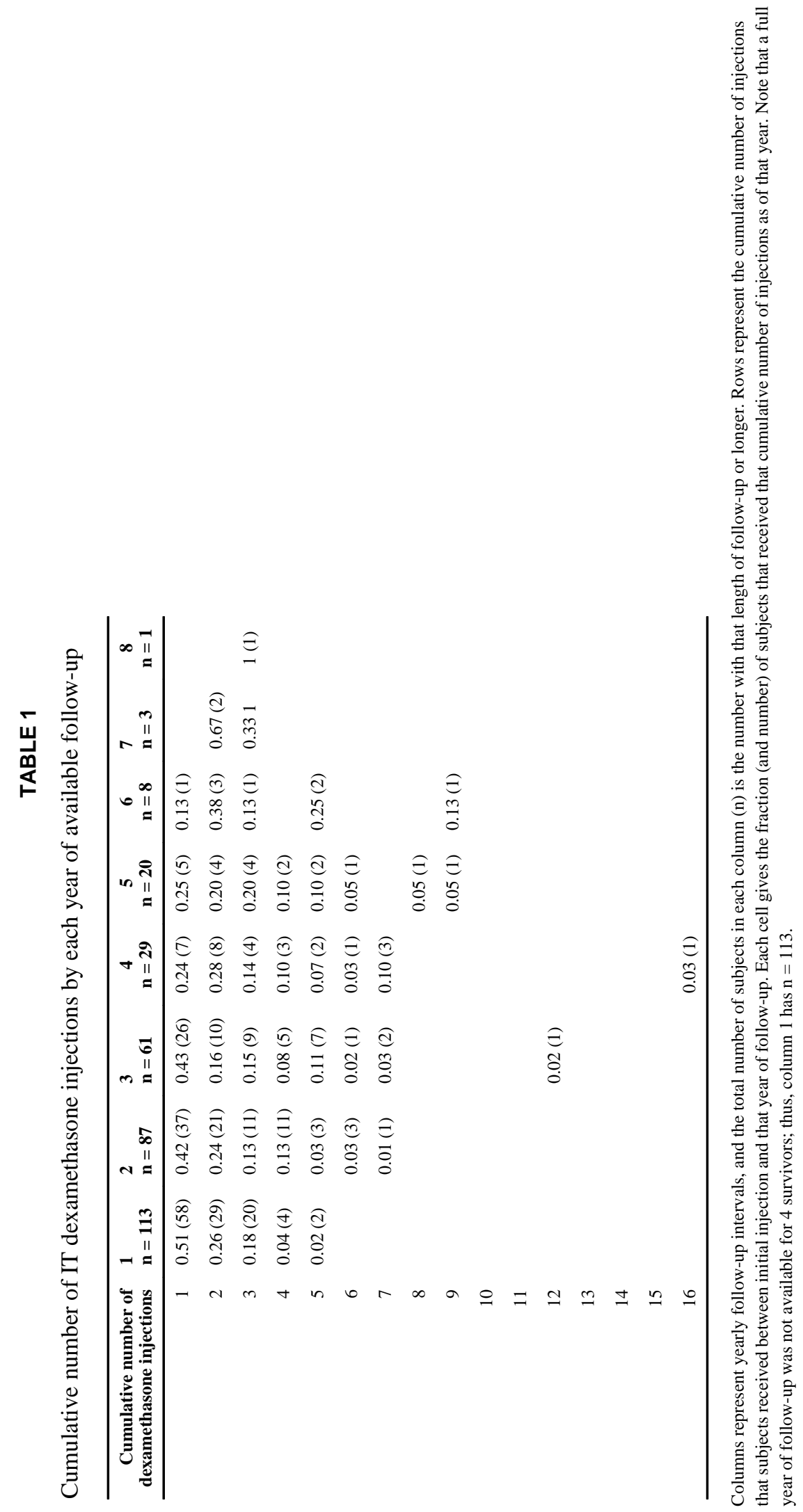

Otol Neurotol. Author manuscript; available in PMC 2010 September 13. 


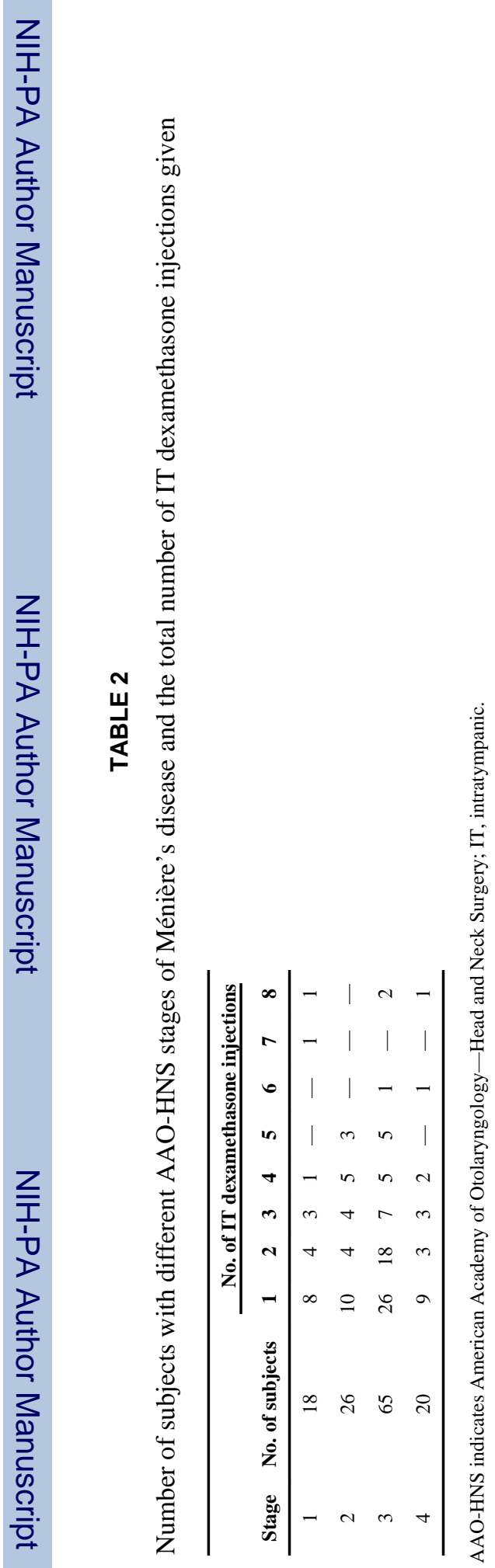

Otol Neurotol. Author manuscript; available in PMC 2010 September 13. 